\title{
Procedure Agents Link Identifier
}

National Cancer Institute

\section{Source}

National Cancer Institute. Procedure Agents Link Identifier. NCI Thesaurus. Code C162023.

A sequence of characters used as a linkage between related procedure agents identifier records. 